\title{
Filament Channels: Essential Ingredients for Filament Formation
}

\author{
V. Gaizauskas \\ Herzberg Institute of Astrophysics, National Research Council of Canada, \\ 100 Sussex Drive, Ottawa, K1A OR6 Canada
}

\begin{abstract}
Channels share with their filaments in a hemispheric dependance for the orientation of their axial magnetic fields. This property has significant implications for models of filaments. In support of new model-building exercises, this review examines known signatures of channels, presents evidence that emerging flux plays a key role in creating a new filament channel, and compares channels which form in the belts of active latitudes with those formed closer to the poles.
\end{abstract}

\section{Introduction}

Solar filaments form in filament channels, seen in the cool chromosphere as long narrow voids overlying polarity inversions between plages of opposite magnetic polarity - hence 'plages couloirs' (Martres et al. 1966). Spicules or fibrils rooted in enhanced magnetic elements are suppressed, even absent, inside channels. Foukal (1971) concluded that the characteristic orientation of $\mathrm{H} \alpha$ fibrils to either side of a filament channel denotes a magnetic field with a predominantly horizontal component along, and not across, the axial direction of the channel. The dominant horizontal direction of fields in filaments themselves was confirmed by the observations of many prominences at the limb using the Hanle effect (reviewed by Leroy 1989).

Radiation originating in hotter plasma is characteristically weakened above polarity inversions with filaments or filament channels - as in He I 10830 (Harvey 1996) or at $\mathrm{mm}$ wavelengths (Bastian et al. 1993), or is completely absent - as in the UV (Dere et al. 1986) or in X-rays (Serio et al. 1978) where the filament channel is equated to the filament cavity (Engvold 1994; Moses et al. 1994). The relationship between these radiative signatures and those revealed by the morphology of the underlying chromosphere is unclear.

Not every filament channel contains a filament, nor does every polarity inversion develop an associated channel. Convergence of magnetic flux, leading to flux cancellation at a polarity inversion, is a necessary condition for producing a filament channel and filament (Martin 1990).

Martin et al. 1994 discovered a hemispheric asymmetry for quiescent filaments and their underlying channels: when quiescent filaments/channels are viewed from the positive polarity side, a majority in the northern (southern) hemisphere have fields directed to the right (left). Thus the filament/channel field has a definite orientation - either "dextral" or "sinistral" - which predom- 
inates in the northern and southern hemispheres, respectively. Channels with a common orientation can join to form very long and very persistent structures. Channels can exceed in length and duration any filament which forms in them.

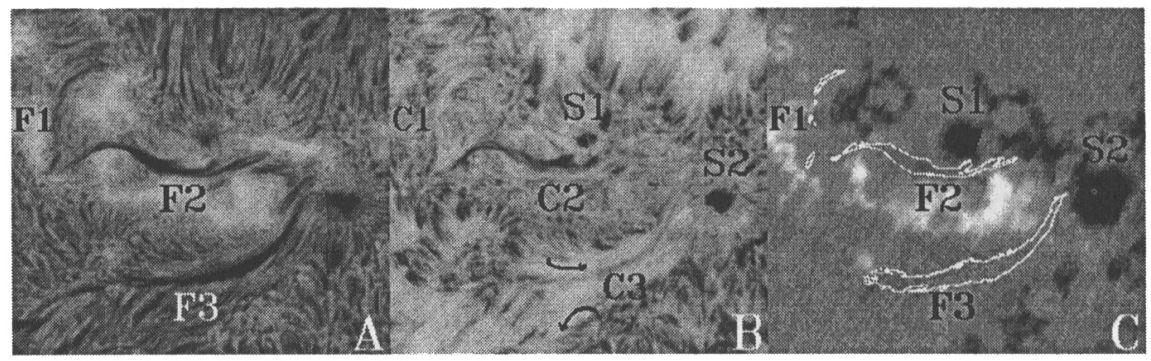

Figure 1. Filament channels in McMath 14726 on April 18, 1977: (a) $\mathrm{H} \alpha$ filtergram; (b) cospatial filtergram at $\mathrm{H} \alpha+0.6 \AA$ with voids between plages at channels $\mathrm{C} 1, \mathrm{C} 2, \mathrm{C} 3$; (c) cospatial NSO/KP B magnetogram with contours of 3 filaments superposed. S1 and S2 are both $p$-polarity spots (black is negative). Top $\mathrm{N}$; right $\mathrm{W}$ in all figures.

Polarity inversions and the channels that outline them are basic ingredients of the Sun's global magnetic topology. McIntosh $(1972,1992)$ used $\mathrm{H} \alpha$ images for two decades to locate channels and to follow their evolution against a backdrop of continually evolving active regions. Filament channels may be implicated in coronal mass ejections (CMEs). Huge soft X-ray arcades, seen with the Yohkoh SXT to form suddenly over extended polarity inversions, followed: (i) a CME in the absence of a filament eruption (McAllister et al. 1996); (ii) 'X-ray blowouts' at polarity inversions near the borders of coronal holes but without any corresponding chromospheric disturbance (Bhatnagar 1996).

This review presents data which show that the visibility of channels depends on local field strengths, that emerging flux plays a key role in creating a new channel, and that channels in the belts of active latitudes are related to those formed closer to the poles.

\section{Signatures of Filament Channels}

The Ottawa River Solar Observatory (ORSO) acquired many large-scale images of filaments and filament channels in raster scans, made daily whenever possible from 1978 to 1992 , of the entire Sun with a $0.25 \AA$ bandwidth $\mathrm{H} \alpha$ filter displaced $0, \pm 0.6 \AA, \pm 1.0 \AA$ at each of 102 overlapping positions on the disc. Images at \pm $0.6 \AA$ are especially useful to detect voids in fibril patterns and to find reversals in the streaming direction of fibrils on opposite sides of a polarity inversion basic signatures of a chromospheric filament channel. Samples presented here from the ORSO archive illustrate a range of channel environments. 


\subsection{In Active Latitudes}

The clearest channels are located on the boundaries of active regions where a spot and plage, evolving together as a unit of bipolar flux, are separated by a polarity inversion from pre-existing flux. Of three filaments in an activity complex comprising two closely packed bipoles (Figure 1a), two have channels (C1 and C3, Figure 1b) at polarity inversions isolating the flux inside the complex from surrounding plages. They are wider than channel $\mathrm{C} 2$ which separates older $p$-polarity spot S1 from the $f$-polarity plage associated with its younger $p$-polarity neighbor, S2 (Figure 1c). Contrary directions of streaming fibrils on opposite sides of broad channel C3, indicated by arrows in Figure 1b, brand C3 as a sinistral channel. $\mathrm{C} 3$ is less voided than $\mathrm{C} 1$ or $\mathrm{C} 2$ : besides the still-visible spine of the filament, faint dark threads cross C3 diagonally in Figure 1b.

Activity complexes are at times spaced almost regularly around the active belts of the Sun (Gaizauskas et al. 1983); polarity reversals between adjacent complexes, typically inclined diagonally across the belt of active latitudes, are often marked by the long quiescent filaments called the 'intermediate' type. Figure 2 compares in the same field of view an intermediate filament near the $\mathrm{E}$ limb with a boundary filament surrounding active region (AR) 7163, a socalled 'anemone' region in an adjacent coronal hole. The void in the channel of the intermediate filament is not yet evident at a wavelength offset of $+0.4 \AA$ (Figure 2a). Instead, numerous low-lying fine dark structures inside the channel are coaligned with the axis of the filament - not across it - over its entire length, similar to the axial alignment of $\mathrm{H} \alpha$ fibrils seen alongside filaments in the core of the line in strong channels (F3 in Figure 1a and F1 in Figure 3). For the boundary filament of AR 7163 , however, a void is already evident at this wavelength (Figure 2b).

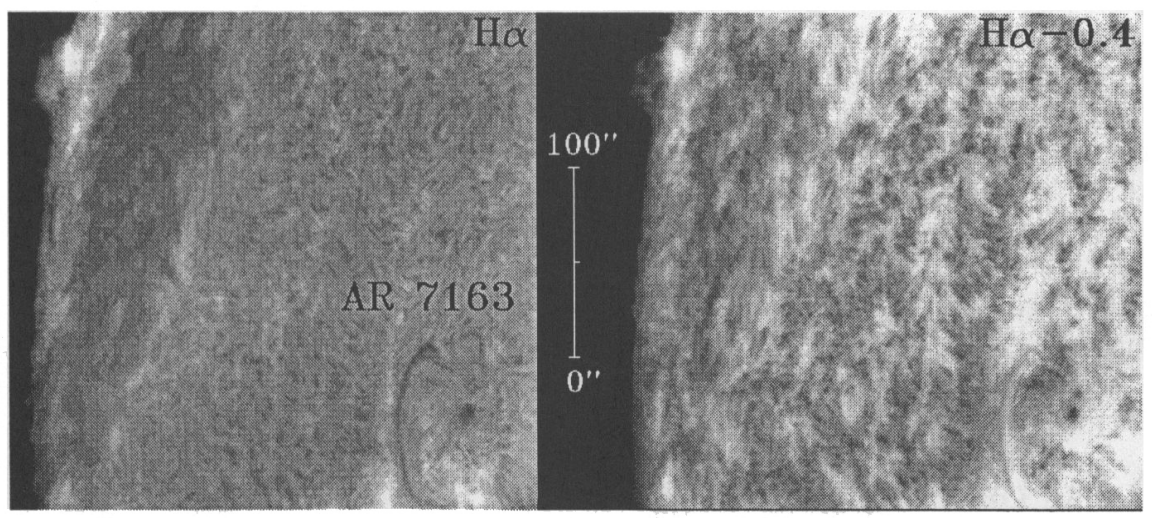

Figure 2. Intermediate and boundary flaments on May 10, 1992.

\subsection{In Quiet Equatorial Regions}

Of three filaments depicted on the same day in Figure 3 only the channel for F1 is obvious, from: coaligned $\mathrm{H} \alpha$ fibrils next to the $\mathrm{H} \alpha$ filament; a strong void at 


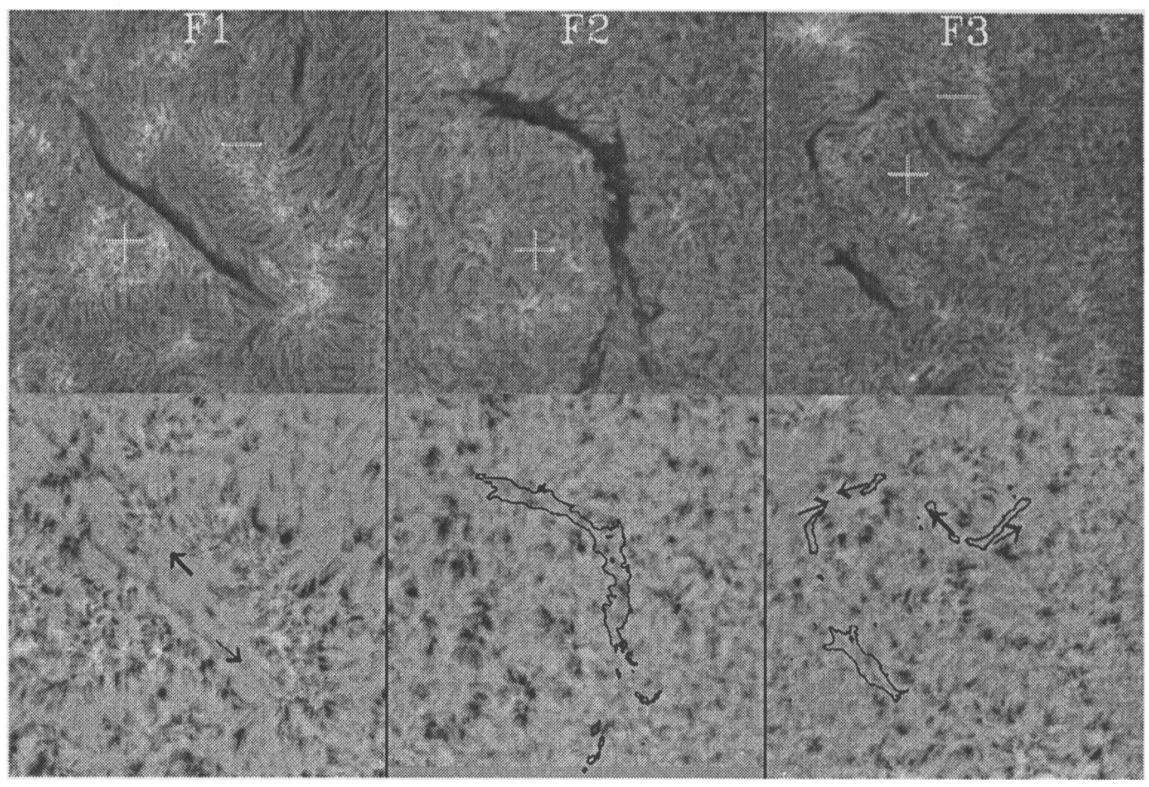

Figure 3. Upper row (filaments) $\mathrm{H} \alpha$ filtergrams; lower row (channels) $\mathrm{H} \alpha+0.6 \AA$ filtergrams with filaments superposed as black contours. ORSO images on July 24,1992 . +/- signs denote magnetic polarity.

$\mathrm{H} \alpha+0.6 \AA ;$ and counter-streaming fibrils on the edges of the channels (black arrows) which brand F1 as a dextral filament. It was stable during its disk passage.

All of these signs are absent for multi-barbed F2; most of its many barbs indicate that F2 is sinistral. The lack of a channel may be related to the fact that F2 altered markedly each day and vanished before reaching central meridian. A channel is barely traceable at fragmented filament F3 from counter-streaming fibrils marked by arrows in the coaligned $\mathrm{H} \alpha+0.6 \AA$ filtergram.

These 3 filaments are superposed as contours against the full-disk SXT image in Figure 4. The arcade of loops overlying dextral filament F1 is a stable feature during this disk transit. Coronal loops over either F2 or F3 are invisible. Only the filament lying between the strongest magnetic fields has both a chromospheric void and an arcade visible with these exposures of Yohkoh/SXT.

\subsection{In Quiet Regions at High Latitude}

Channels for polar crown filaments (PCFs) are indistinct or invisible in $\mathrm{H} \alpha$. Sub-polar crown filaments, which form along the high-latitude fringe of active latitudes, can have conspicuous $\mathrm{H} \alpha$ channels. Both kinds were present in July 1979 (see Figure 1 of Harvey and Gaizauskas 1998, these proceedings) as a single long structure outlining a large-scale unipolar pattern of negative (trailing) polarity flux transported poleward from the northern belt of active latitudes and sheared by differential rotation, to form a 'switchback'. This channel is remark- 


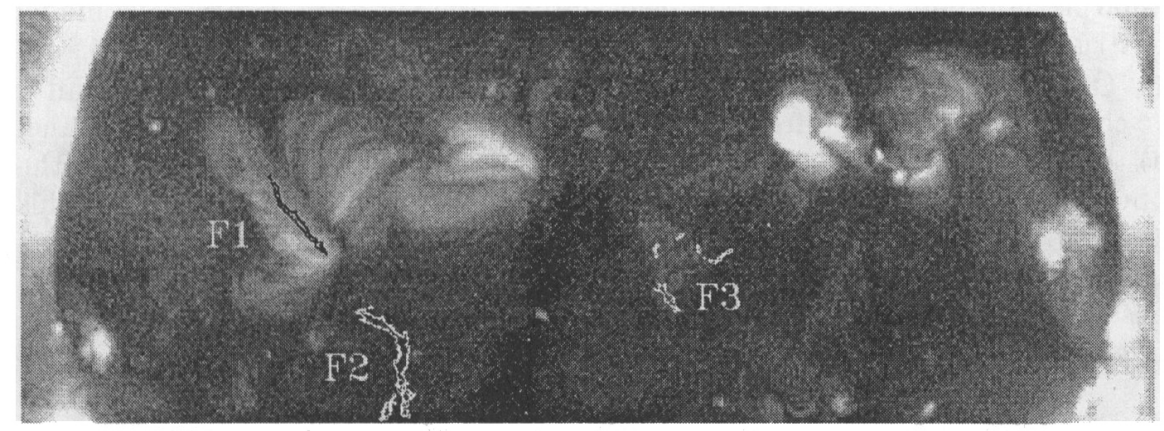

Figure 4. SXT(Yohkoh) image for July 24, 1992 with $\mathrm{H} \alpha$ filaments superposed as black (F1) or white (F2, F3) contours.

ably continuous in the He I 10830 image as a path with weakened absorption and reduced contrast in the network. Fragments of filaments overhang the entire channel. In the polar crown portion, $\mathrm{H} \alpha$ fibrils lack systematic alignments; but in the sub-polar crown portion, many $\mathrm{H} \alpha$ fibrils are axially aligned along the axis of the channel.

In summary, $\mathrm{H} \alpha$ channels are clearest (anti-parallel fibrils, clear voids) at polarity inversions embedded in strong magnetic fields; they are vague in weak fields. Where magnetic fields are weakest, as in polar regions, He I 10830 remains a sensitive indicator of filament channels.

\section{Creation of Filament Channels}

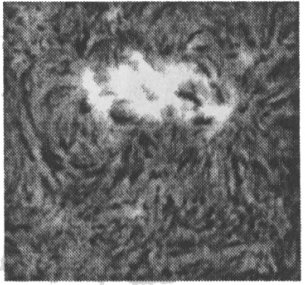

(a)

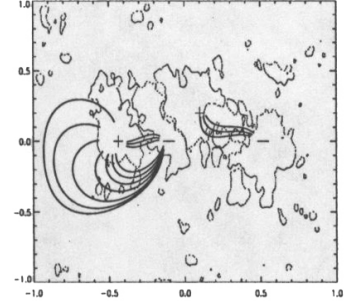

(b)

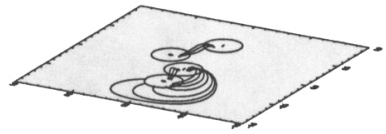

(c)

Figure 5. Field lines in a filament channel (Courtesy D.H. Mackay)

It follows that the best place to find an $\mathrm{H} \alpha$ filament channel in the act of formation should be among strong fields. A successful search for such an event is described by Gaizauskas et al. (1997). Because flux emerged in an undisturbed background, it was possible to trace the detailed evolution of $\mathrm{H} \alpha$ fibrils surrounding the new small activity complex. A channel formed in a few hours but a stable filament did not form in it for another 4 days. Key to the formation of the channel was the close emergence of two bipoles, not one alone, resulting in 
a sharply non-potential magnetic field close to the surface. A force-free model constructed by Mackay et al. (1997) for this example is shown in Figure 5.

On the left side of Figure 5a the aligned coarse fibrils are seen a day after the channel formed; force-free field lines modeled for $\alpha=3.0$ are plotted in panel (b) to show their close fit to the direction of the $\mathrm{H} \alpha$ fibrils in the channel; the oblique view in panel (c) emphasizes how flat and shallow the field lines are in this situation. The filament forms when flux from the newly arrived concentration of flux converges onto an old diffuse region and the magnetic connectivity of field lines in the channel changes due to magnetic reconnection (see Galsgaard and Longbottom, these proceedings).

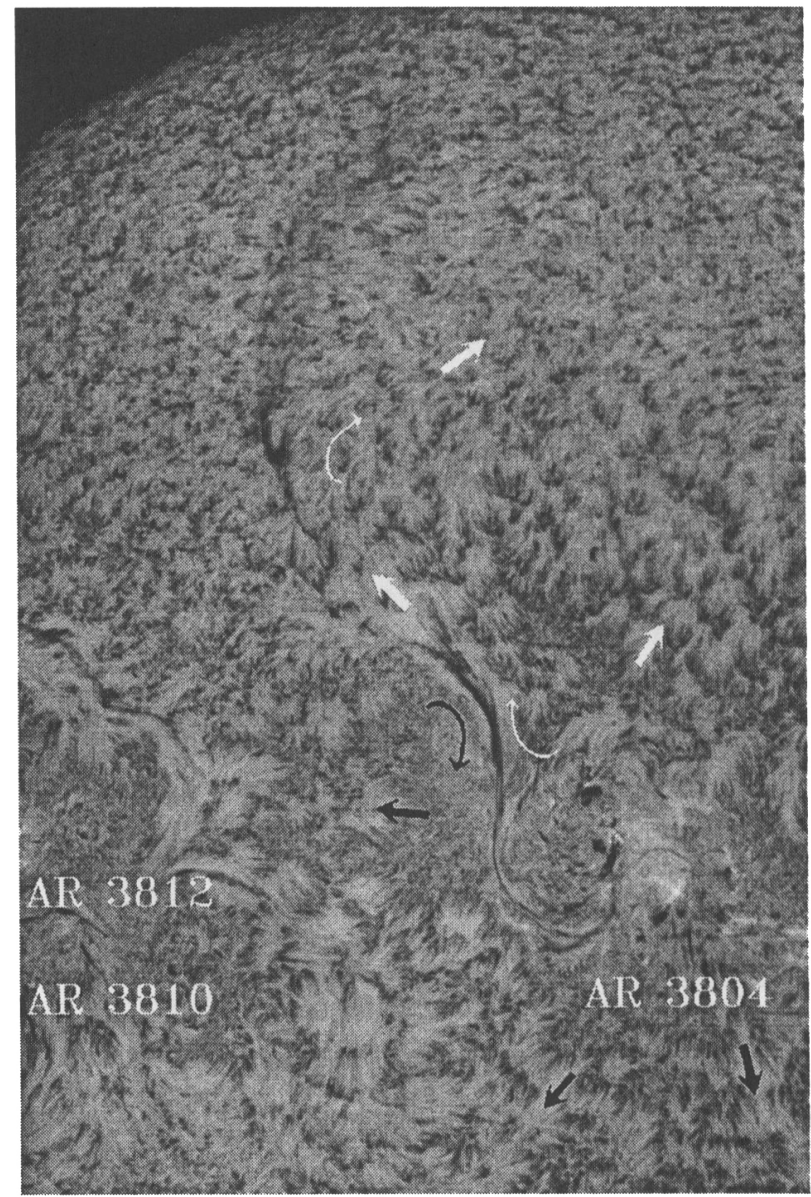

Figure 6. Sunspot filament in AR 3804 on July 15, 1982. Fibrils stream in directions of arrows: white/black $=+1$ - polarity. Mosaic of 15 ORSO images at $\mathrm{H} \alpha+0.6 \AA$.

Filaments inside activity complexes are at one extreme of flux concentration. Figure 6 shows one anchored in the large $\delta$-type spot of the most flare-active 
region of Cycle 21, AR 3804, within a long-lived ( $\geq 20$ solar rotations) complex of activity. The channel is defined by anti-parallel fibrils (between the curved black and white arrows) at the edges of enhanced network on opposite sides of the void partially filled with a strongly Doppler-shifted segment of the filament. Fibrils are also aligned over a much broader area radiating away from AR 3804 and away from the polarity inversion containing the long filament. From their directions we can infer that a huge arcade overlies the narrow portion of the filament. The randomly oriented fibrils underneath the broad northern portion of the filament give no hint there of a channel or an overlying arcade.

The enhanced network arose from the dispersion of earlier flux emergences in the same activity complex. In two more rotations, enhanced network of both polarities had migrated up to the coronal hole covering the polar cap. During that migration, the filament channel depicted in Figure 6 first elongated and then fragmented as the supply of flux in the core of the activity complex weakened. By September 1982 the filament and channel of Figure 6 had disappeared; a newer and longer one had formed between the fluxes diffusing poleward from the complex containing AR 3804 and from its eastern companion, AR 3810 and AR 3812 combined (Figure 6). Furthermore, the huge switchback formed by these rapidly evolving patterns now had fragments of a PCF between the advancing enhanced network and the polar coronal hole. The steps - major flux eruption to formation of a PCF - were completed in just 3 or 4 solar rotations.

\section{Discussion}

A relationship between chromospheric voids in channels and overlying coronal cavities has been long suspected (Section 1). A recent Yohkoh/SXT observation at the limb (Figure 2 of Harvey and Gaizauskas, these proceedings) supports this conjecture. An exceptionally sharp outer boundary of an X-ray cavity terminates precisely at the outer edges of a channel defined by a void in $\mathrm{He}$ I 10830 enclosing a filament. This is the first direct evidence that a 'coronal' cavity, terminating at the surface, could result in reduced density surrounding a filament at chromospheric heights. The reduced visibility in channels of off-band $\mathrm{H} \alpha$ and $\mathrm{He}$ I 10830 fine structure may thus be due simply to a lack of sufficient matter to illuminate low-lying field lines at these wavelengths. This suggestion is consistent with the increasing visibility reported in Section 2 of chromospheric voids with increasing magnetic flux density surrounding channels, which in turn is consistent with the findings about coronal X-ray arches and filament channels by Davis and Krieger (1982). They found that the arches were brightest above filament channels with little or no filamentary matter in strong fields on the fringe of active regions, but were absent above strongly absorbing filaments located beneath $\mathrm{X}$-ray cavities typical for quiescent filaments.

A density reduction alone in the channel cannot account for the He I 10830 signature in the absence of an $\mathrm{H} \alpha$ one in large quiescents such as PCFs. Coronal cavities above these tall structures reduce the downward flux of radiation and hot electrons, thereby weakening the He I 10830 absorption in the channel. Any axial field in the filament will further inhibit downward thermal conduction.

The behaviour of PCFs with the solar cycle has often been represented as a secular trend and linked to the solar dynamo (review by McIntosh 1992). 
The preliminary case study based on Figure 6 shows instead that PCFs and their channels can occur in a highly episodic fashion and are more likely driven by the transport to high latitudes of large quantities of surface flux originally concentrated in activity complexes at much lower latitudes.

Acknowledgments. I thank T. Forbes, K. Harvey, D. Mackay, and C. Zwaan for stimulating discussions on channel problems. Presentation of this work at IAU Colloquium 167 in coordination with co-investigators was supported by NSF Grant ATM-9696256. K. Harvey kindly transferred images from NSO/KP and the Yohkoh/SXT. The NSO/KP data are produced cooperatively by NSF/NOAO, NASA/GSFC, and NOAA/SEC. The Yohkoh satellite is a project of the Institute of Space and Astronautical Sciences, Japan.

\section{References}

Bastian, T.S., Ewell, M.W., Jr., and Zirin, H. 1993, ApJ, 418, 510

Bhatnagar, A. 1996, in Solar and Interplanetary Transients, (eds.) S. Ananthakrishnan and A. Pramesh Rao, Astrophys. and Space Sci. Vol. 243, Kluwer Acad. Publ., Dordrecht, Holland

Davis, J.M. and Krieger, A.S. 1982, Solar Phys., 80, 295

Dere, K.P., Bartoe, J.-D.F., Brueckner, G.E. 1986, ApJ, 305, 947

Engvold, O. 1994, in Solar Coronal Structures, (eds.) V. Rusin, P. Heinzel, and J.-C. Vial, VEDA, Slovakia, p. 207

Foukal P. 1971, Solar Phys., 19, 59

Gaizauskas, V. et al. 1983, ApJ, 265, 1056

Gaizauskas, V., Zirker, J.B., Sweetland, C., and Kovacs, A., 1997, ApJ, 479, 448

Harvey, K.L. 1996, in Solar Wind Eight, (eds.) D. Winterhalter et al., AIP Conf. Proc. 382, AIP:Woodbury, NY, p. 9

Leroy, J.L. 1989 in Dynamics and Structure of Quiescent Prominences, (ed.) E.R. Priest, Kluwer:Dordrecht, p. 77

Mackay, D.H. et al. 1997, ApJ, 486, 534

Martin, S.F. 1990, in Dynamics of Quiescent Prominences, (eds.) V. Ruzdjak and E. Tandberg-Hanssen, Springer:Berlin, p. 1

Martin, S.F., Bilimoria, R., and Tracadas, P.W. 1994, in Solar Surface Magnetism, (eds.) R. Rutten and C. Schrijver, Dordrecht:Kluwer, p. 303

Martres, M.-J., Michard, R., and Soru-Iscovici, I. 1966, A\&A, 29, 249

McAllister, A.H. et al. 1996, J. Geophys. Res., 101, 13,497

McIntosh, P.S. 1972, Rev. Geophys. and Space Sci., 10, 837

McIntosh, P.S. 1992, in The Solar Cycle, (ed.) K.L. Harvey, ASP Conf. Ser. Vol. 27, p. 14

Moses, D. et al. 1994, ApJ, 430, 913

Serio, S. et al. 1978, Solar Phys., 78, 65 